\title{
Telogen Effluvium
}

National Cancer Institute

\section{Source}

National Cancer Institute. Telogen Effluvium. NCI Thesaurus. Code C112200.

A scalp hair loss condition characterized by excessive shedding of hair in the resting phase of growth, usually following a fever or major body stress. 